\title{
A comprehensive guide to telocytes and their great potential in cardiovascular system
}

\author{
Kucybala $\mathrm{I}^{1}$, Janas $\mathrm{P}^{1}$, Ciuk $\mathrm{S}^{1}$, Cholopiak $\mathrm{W}^{1}$, Klimek-Piotrowska $\mathrm{W}^{2,3}$, Holda $\mathrm{MK}^{2,3}$ \\ Faculty of Medicine, Jagiellonian University Medical College, Cracow, Poland. mkh@onet.eu
}

\begin{abstract}
Telocytes, a recently discovered type of interstitial cells, have a very distinctive morphology - the small cell body with long extensions, named telopodes. In our review, apart from introducing general aspects of telocytes, we focus on properties, functions and future potential of those cells in cardiovascular system. However, physiological functions of telocytes in cardiovascular system are still regarded as quite enigmatic. Previous studies claim that they play a role in organogenesis and regeneration, bioelectrical signalling, mechanoelectrical coupling, anti-oxidative protection, angiogenesis and regulation of blood flow. As well, they are presumably connected with the presence of blood-myocardium barrier and proper organisation of extracellular matrix. Moreover, there exists a significant link between the quantity of telocytes in tissue and numerous cardiovascular diseases such as: myocardial infarction, cardiomyopathies, systemic sclerosis, heart failure, atrial fibrillation, isolated atrial amyloidosis, myxomatous valve degeneration and hyperplastic consequences of vascular injury. Thanks to their unique properties, telocytes might be a breakthrough in treatment of cardiovascular diseases, as they may be effective in reversing effects of myocardial infarction. Telocytes also may play a major role in tissue engineering - they might be the key factor in creating stable and efficient vascular network in larger synthetic tissues or organs (Tab. 1, Fig. 3, Ref. 53). Text in PDF www.elis.sk.

KEY WORDS: telocytes, heart, blood vessels, cardiovascular system, cardiovascular diseases, regenerative medicine.
\end{abstract}

\section{Introduction}

Telocytes (TCs) are identified as a new cell type among interstitial cells. The main feature that distinguishes TCs from any other cells are their very long and thin prolongations, which are named telopodes (Tps) (1). The history of their development derives in a straight line from Interstitial Cajal Cells (ICC).

\section{A brief history}

ICC have been described for the first time by a Spanish neuroanatomist, Nobel laureate - Santiago Ramon y Cajal in 1889 (1). He found single, tiny ganglionic cells in the specimens from mammalian gastrointestinal tract and named them as interstitial neurons. On the turn of the 70's and 80's, electron microscopy of the human oesophagus and stomach proved the existence of those cells in gastrointestinal tract. At that point the concept of their neuronal origin was overthrown and mesenchymal one came to light (2).

In 2005 Popescu et al, using electron microscopy and Cajal's staining, found interstitial cells, which were similar to ICC in the

${ }^{1}$ Faculty of Medicine, Jagiellonian University Medical College, Cracow, Poland, ${ }^{2}$ Department of Anatomy, Jagiellonian University Medical College, Cracow, Poland, and ${ }^{3}$ HEART - Heart Embryology and Anatomy Research Team, Jagiellonian University Medical College, Cracow, Poland Address for correspondence: M.K. Holda, Department of Anatomy, Jagiellonian University Medical College, 12 Kopernika Street, 31-034 Cracow, Poland. Phone: +0048.12 .4229511$ specimens from the mammary gland. They initially called them Interstitial Cajal-like Cells (ICLC) (3). After that discovery Pieri et al re-examined the muscular coat of the gut only to find out that ICLC are present there in great quantities, coexisting with the ICC and often share an identical distribution (4). The conclusion was - those are two different types of cells. Furthermore, in 2010 Popescu et al suggested that ICLC should change their name to clearly emphasize the separateness of these two cell populations. As a consequence, the word "telocytes", was suggested as a new name-from a Greek word "telos", which refers to the object of huge potential (by Aristole) (1).

Considering TCs' concept long road to full acceptance, where all steps of exploration were accompanied by the development of new, more precise methods of detection and differentiation, they ought to await bright investigational future. TCs now seem to be an interesting subject of study for representatives of almost all clinical fields. With their huge likelihood of usage in the understanding and management of diseases of cardiovascular system, we decided to review the up-to-date accomplishments in this field. We also decided to present the prospect of probable direction of development of their use in the future.

\section{Telocytes in general}

\section{Cell morphology}

TCs have small body cells with $1-5$ (predominantly $2-3$ ) very long $(10-1000 \mu \mathrm{m})$, thin Tps containing dilatations termed podoms 


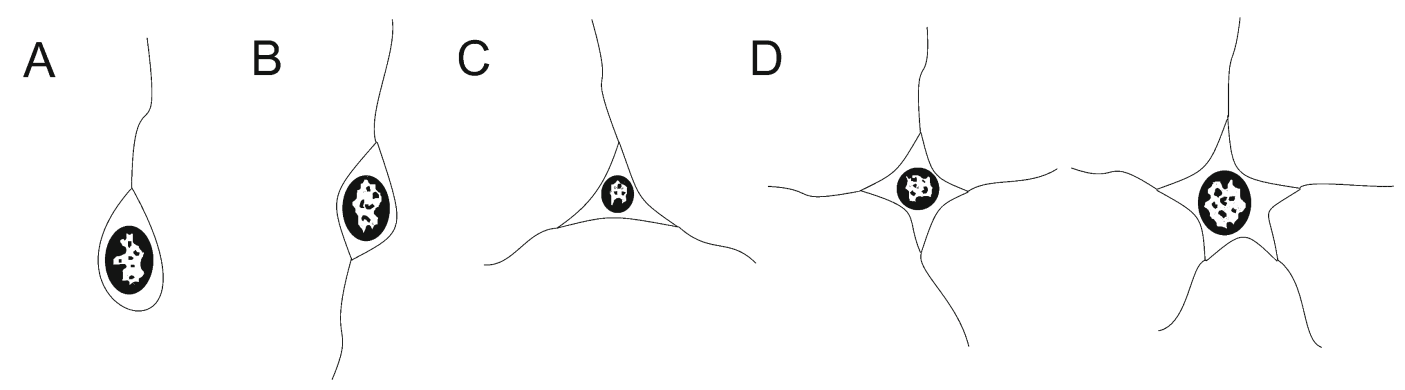

Fig. 1. Shapes of telocytes depending on the number of telopodes. A - piriform, B - spindle, C - triangular, D - stellate.

and thin fragments named podomeres (5). The presence of Tps is the most characteristic feature of TC, which differentiate TCs from other known cells. Except for neuronal axons, Tps are probably the longest structures in the body. Intermittently, Tps may be branched dichotomously, creating distinctive pattern $(1,6)$.

The shape of a TC's cell body depends on the number of Tps. The TC with one Tp is piriform-shaped, with two - spindle, three - triangular, four and more Tps - stellate (Fig. 1) (1). Cell body comprises large oval nucleus occupying about $25 \%$ of cell volume with peripheral heterochromatin rim, small amount of cytoplasm, mitochondria, small Golgi apparatus, smooth and rough endoplasmic reticulum and lipid blobs (1). Podoms contain numerous mitochondria, caveolae, coated vesicles and well developed rough and smooth endoplasmic reticulum $(6,7)$. Microtubules, intermediate (mostly composed of vimentin) as well as thin filaments could also be observed inside of Tps (6).

\section{Location}

TCs are located in the various organs, both in normal and altered tissues. So far, they have been examined in: heart, blood vessels, thoracic duct, trachea and lungs, pleura, trigeminal ganglion, meninges, choroid plexus, dura mater, urinary system, uterus, oviduct, mammary gland, placenta, testicles, prostate, parotid gland, minor salivary glands, gastrointestinal tract, mesentery, pancreas, liver, gallbladder, spleen, eye, skin, fascia lata, temporomandibular joint disc, skeletal muscles, neuromuscular spindles, bone marrow $(5,8,9)$. They were also discovered in cancerous tissues: squamous cell carcinoma and basal cell carcinoma, as well as in mammary gland carcinoma (5).

\section{Identification of TCs}

The most common methods used for TCs' identification are: transmission electron microscopy (TEM), flow cytometry com-

Tab. 1. Differences in expression of significant markers between telocytes (TCs), fibroblasts, pericytes and neurons (18-21).

\begin{tabular}{lcccc}
\hline & TCs & Fibroblasts & Pericytes & Neurons \\
\hline CD34 & + & - & - & - \\
c-kit & + & - & - & $+/-$ \\
$\alpha-S M A$ & $+($ weak $)$ & - & + & $\mathrm{n} / \mathrm{d}$ \\
PDGFR- $\beta$ & + & + & + & + \\
Vimentin & + & + & + & + \\
\hline
\end{tabular}

$\mathrm{n} / \mathrm{d}$ - no data available bined with specific antibodies and immunofluorescence staining $(5,7)$. In TEM, which is a gold standard for identifying TCs, they are defined as cells with Tps and other cellular structures typically located in cell body, presenting specific morphology (see above), which are located in interstitium, in extraepithelial space, between functional elements (for instance nerves or arteries) $(5,10)$.

Immunophenotype of TCs is disparate in different organs and tissues (11). Generally, the most useful markers for TCs identification are CD34 and PDGFR $\alpha$ (12). Other helpful markers are: c-kit, vimentin, $\alpha$-SMA, caveolin-1, iNOS, VEGF, Oct-4, Nanog and Sca-1 $(13,14)$. However, some exceptions exist, for example TCs located in gastrointestinal tract do not express c-kit (15). TCs' immunophenotype also changes during their life cycle. During embryonic life TCs are c-kit and CD34 negative (16). Additionally, Chang et al proved that intensity of c-kit, Sca-1 and Nanog expression varies depending on the sub-cellular localisation. C-kit is strongly expressed in cell bodies and detectable in Tps, while Sca-1 can be found in cell bodies and in the proximal parts of Tps, on the other hand Nanog is expressed only in cell bodies (13).

\section{The similarity to other cells}

It is difficult to discriminate TCs from other cells presenting similar phenotype, like fibroblasts, pericytes and neurons, only with use of TEM. Immunofluorescence staining is a useful method in this case (17). The most appropriate marker used for differentiating TCs from those cells is CD34. In contrast to TCs, fibroblasts, pericytes and neurons are CD34 negative. Nevertheless, CD34 is not a perfect marker, because endoneurial fibroblasts are also CD34 positive, similarly to TCs (5). Differences in expression of other significant markers between TCs, fibroblasts, pericytes and neurons are shown in Table 1 (18-21).

Moreover, telomerase activity is significantly lower in cardiac TCs than in fibroblasts (18). Another difference between those cells concerns the expression of miR-193; TCs do not express miR-193, contrary to fibroblasts (22).

\section{Intercellular junctions}

TCs create two types of intercellular junctions: homocellular and heterocellular. TCs can also communicate by shedding membrane microvesicles. Additionally, connections with components of extracellular matrix (for instance collagen, elastic fibres) were observed (5). 
302-309

TCs are connected with each other and form a three-dimensional network (23). Homocellular junctions are non-characteristic links made by simple apposition of plasmalemmas of two neighbouring cells (14). They are usually localised between Tps, but intermittently this type of connection was observed between Tp and TC's cell body. TEM imaging showed that TCs are also connected with each other by a few types of adherens junctions: puncta adhaerentia minima, processus adhaerens (which joins overlapping Tps), recessus adhaerens (observed between two Tps and between $\mathrm{Tp}$ and cell body) and manubria adhaerentia (24).

Moreover, TCs link with each other by creating gap junctions (nexus). This type of connection allows to transport ions and small molecules between cells, which might be associated with calcium-dependent mechanism of synchronic release of extracellular vesicles from numerous TCs (25).

It is also proved that TCs create heterocellular junctions with stem cells, nerve cells, fibroblasts, endothelial cells, pericytes, macrophages and mast cells (5). As for the types of connections, stromal synapses, point contacts, nanocontacts and planar contacts were observed $(14,26)$. The most common type of contact between TCs and cardiac stem cells (CSCs) are stromal synapses. Puncta adhaerentia and communication by extracellular vesicles were also observed. Gap junctions between TCs-CSC were not found (26). TCs may link with interstitial cells like fibroblasts, mast cells and macrophages by point contacts and planar junctions and with capillaries by point and planar contacts. Point contacts were also observed between TCs and basal lamina of Schwann cells (24).

TCs may play a significant role in long-distance heterocellular communication by shedding extracellular vesicles (14). The transmission electron microscopy and electron tomography imaging have shown that TCs produce three types of extracellular vesicles: exosomes, ectosomes and multivesicular cargos enclosed by bilayer membrane (27). Thanks to the fact that podoms contain organelles essential for protein synthesis, TCs can produce and send molecular signalling proteins to target cells, which is crucial for differentiation and development of stem cells $(7,25)$. Kostin et al proved that TCs secrete IL-6, VEGF, MIP-1 $\alpha$, MIP-2, MCP-1 and some other chemokines that may affect proliferation and differentiation of CSCs (18). Communication performed by extracellular vesicles probably takes part in modulating homeostasis, stem cells activity, tissue repair, and other complex functions (28).

\section{Cardiac telocytes}

\section{Distribution in the heart}

TCs represent a relatively small fraction of cardiac interstitial cells; their quantity fluctuates between 0.5 to $1 \%$ of all interstitial cells in human heart (29). The number of TCs in human myocardium alternates during lifetime, from $22 \pm 2 \mathrm{TCs} / \mathrm{mm}^{2}$ in newborns and $27 \pm 4 \mathrm{TCs} / \mathrm{mm}^{2}$ in children to $15 \pm 3$ to $19 \pm 3 \mathrm{TCs} / \mathrm{mm}^{2}$ in adults $(29,30)$.

Multiple studies proved presence of three-dimensional spatial network of TCs in many parts of heart, such as: endocardium, myocardium, epicardium, valves and myocardial sleeves of pulmonary veins $(7,31,32)$. However, the distribution of TCs is not homogenous in the whole heart - it is supposed that atria are more abundant in TCs than ventricles, but quantitative data is still missing (6). The deployment of TCs in myocardial layers is also not identical, subendocardially and intramyocardially they form a regular network, but subepicardially they are rather accumulated in clusters in loose connective tissue (6). Moreover, there is a significantly more numerous population of TCs in epicardium than in myocardium (33). Subepicardium and epicardium, in the part close to the coronary artery branching, are the locations of cardiac stem cell niches - the place where CSCs coexist with TCs and many other supporting cells $(10,14,34)$. As for the valves, it is proven that TCs exist in human mitral, tricuspid and aortic valve (35).

\section{Molecular characteristics of cardiac TCs}

Thus far, it is confirmed that cardiac TCs express such molecules as: CD34, c-kit (CD117), PDGFR- $\alpha$ and $-\beta$, vimentin, CD29, Nanog, Sca-1 $(13,18,19)$. CD34, the most reliable marker of TCs, is not uniformly expressed by cardiac TCs from conception to death - in embryonic life TCs are negative for CD34 and after birth they gradually gain CD34 expression $(14,16)$. However, there is still a search for tissue-specific markers of cardiac TCs, which will help determine a specific role of these cells in a human heart (16).

\section{Contacts with other cells}

TCs form heterocellular contacts with a great variety of cells, such as: cardiomyocytes, cardiomyocyte progenitors, cardiac stem cells, endothelial cells, pericytes, Schwann cells, macrophages, mast cells, lymphocytes, fibroblasts/myofibroblasts and valve interstitial cells $(11,18,33,35)$. One of the most distinctive types of TCs-related junctions in heart is a stromal synapse, which links TCs with CSCs and immune cells $(11,26)$. Stromal synapse is the structure in which two cells are within the range of molecular interaction (15-100 $\mathrm{nm}$ ) and a synaptic cleft with electron-dense nanostructures exists between them. In heart, the average length of synaptic cleft between TCs and CSCs ranges from 20 to $30 \mathrm{~nm}$ (26). Other important means of communication between TCs and other cells in heart is shedding extracellular vesicles (exosomes, ectosomes and multivesicular cargo) (27). Extracellular vesicles enable paracrine signalling between TCs and cardiomyocytes, cardiomyocyte progenitors, cardiac stem cells and probably also fibroblasts/myofibroblasts $(11,27,34)$. As paracrine signalling is vital to TCs' functions, it is consistently the vast subject of research. Until now, it has been confirmed that there is a bidirectional exchange of microRNAs between TCs and CSCs, which allows epigenetic signalling and impacts on post-transcriptive mechanisms - in consequence, cells respond faster (36). Additionally, previous studies suggested that cardiac TCs secrete such molecules as: MIP-1 $\alpha$, MIP-2, MCP-1, IL-6, VEGF and NOS2 (37).

\section{Physiological functions of cardiac TCs}

TCs are the key feature of cardiac growth, regeneration and renovation, thanks to their unique communication with cardiac stem cells and progenitors $(7,12)$. Starting from the beginning, TCs have their role in organogenesis of the heart, as they build a 

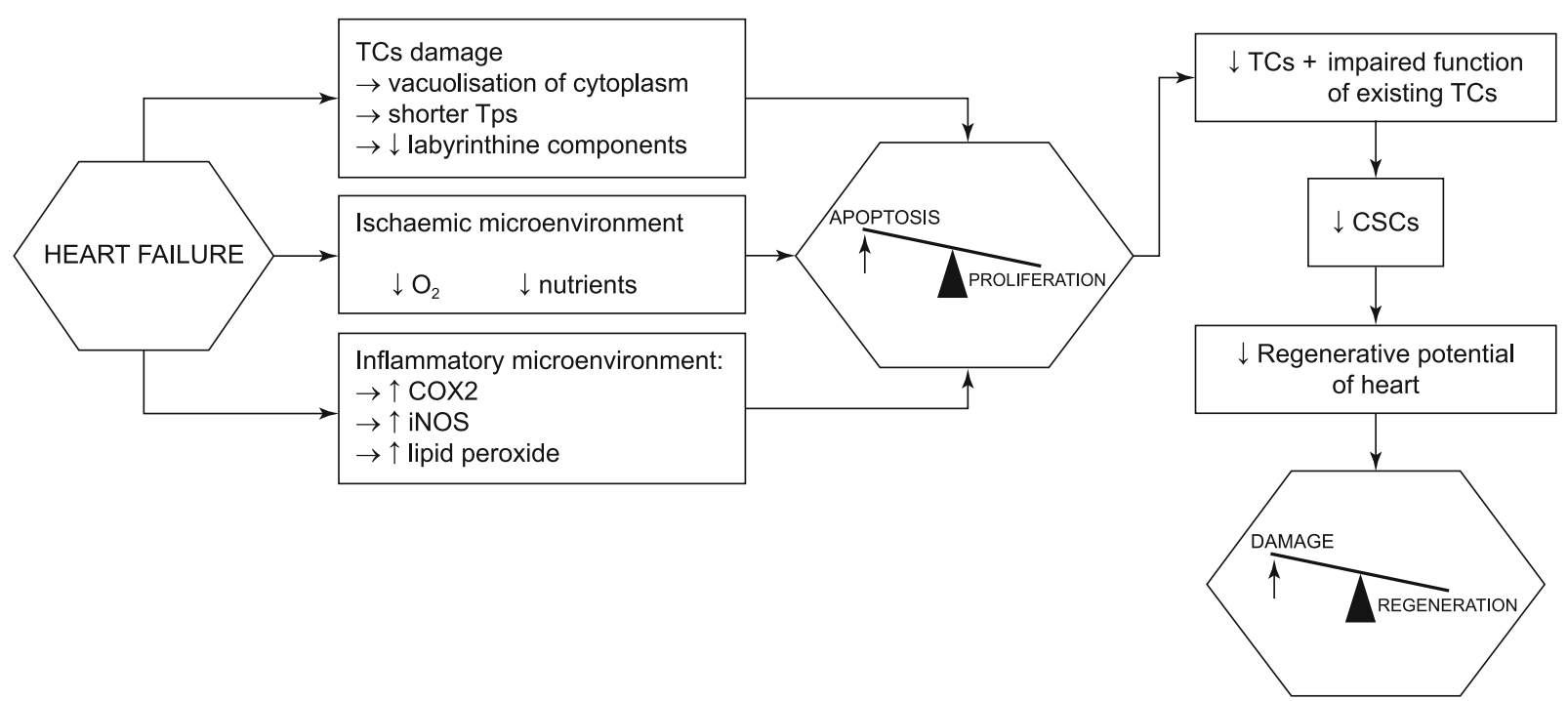

Fig. 2. The mechanism of progressive degeneration of heart in patients with heart failure including the role of telocytes (TCs). CSCs - cardiac stem cells, Tps - telopodes

scaffold in the developing human heart, which guides and nurses cardiomyocyte progenitors during their proliferation and differentiation (12). Similarly, in fish and amphibians after cutting off a part of the heart, the first cells involved in its regeneration are TCs - their bodies and Tps form the network, which guides regeneration of cardiac architecture (29). During the lifetime, TCs play a role of accessory cells in cardiac stem cell niches, where they provide nutrition to stem cells, control their differentiation and help maintain the homeostasis of the niche $(7,11)$. However, along with ageing, decrease in TCs number occurs and regenerative potential of human heart diminishes, but not completely (29). Regenerative capability of an adult human heart still exists; for instance, it could be induced by physical exercise, which activates proliferation of CSCs and TCs (38).

Apart from contacts with stem cells, there is another distinctive property of TCs, which makes them important for sustaining wellbeing of heart tissue. TCs express very high amount of mitochondrial superoxide dismutase (SOD2), which makes them crucial for inhibition of oxidative stress and prevention of cellular aging (39).

As TCs form abundant spatial network interconnecting all components of the heart, they regulate local homeostasis of cardiac tissue and integrate signals from numerous systems, such as vascular, nervous, immune and interstitial $(7,33)$. This attribute of TCs also facilitates long-distance intercellular signalling, including bioelectrical type, which is vital for pacemaking activity of the heart $(14,28)$. TCs express large conductance $\mathrm{Ca}^{2+}$-activated $\mathrm{K}^{+}$current $\left(\mathrm{BK}_{\mathrm{Ca}}\right)$ and inwardly rectifying $\mathrm{K}^{+}$current $\left(\mathrm{IK}_{\mathrm{ir}}\right)$, which along with mechanical integrity of the network probably play a role in mechanoelectrical coupling during heart contractions, resulting in synchronous contractures of cardiomyocytes (40).

In addition, cardiac TCs act as an important component of connective tissue in heart, as they are crucial for maintaining normal organisation of extracellular matrix (38). Based on the example of heart valves, TCs' network helps in balanced force distribution in tissue and enables to maintain proper valve flexibility (35). TCs are also supposed to be a source of cardiac mesenchymal cells, because they express mesenchymal marker CD29 and have the ability to lose expression of CD34 (19).

TCs closely surround one third of endothelial cells in subendocardial tissue and thus, there is an assumption that TCs may be prerequisite component of endocardial blood-myocardium barrier (31).

\section{Role of cardiac TCs in pathological conditions}

It is well established that pathological processes within the heart are associated with a decreased number of TCs (18). Among them, myocardial infarction (MI) can be mentioned. TCs are particularly prone to ischaemia and hence their network is almost completely destroyed due to MI (41). Other conditions connected with TCs depletion are dilated, ischaemic and inflammatory cardiomyopathies as well as cardiac manifestation of systemic sclerosis $(15,30)$.

Fibrosis, a consequence of many pathological states in a human heart, is closely related with the number of TCs and their Tps in heart tissue (15). An increase in fibrillary collagens as a result of replacement fibrosis is correlated with the reduction of TCs and Tps quantity or even their absence $(18,30)$. It is supposed that TCs may have an impact on fibrosis thanks to direct contacts and paracrine signalisation between them and fibroblasts/ myofibroblasts, but their role has not been fully clarified yet (11). However, based on the example of systemic sclerosis, there exists a theory that the loss of TCs results in increased fibroblast-tomyofibroblast conversion and allows further spreading of elastin and collagen fibres in tissue (15).

All previously mentioned pathologies linked with the decrease in TCs number imminently lead to end-stage disease - heart failure. A characteristic manifestation of TCs degeneration in heart 
failure is described as vacuolisation of cytoplasm, shrinkage of Tps, lack of labyrinthine components along with inflammatory or ischaemic micro-environment, rich in iNOS, COX-2 and lipid peroxide (18). As well, TUNEL labelling proved imbalance between TCs proliferation and apoptosis - with the domination of the latter. In consequence, TCs' ability to maintain cardiac stem cell niche is impaired - it provokes depletion of CSCs (30). Thus, regenerative potential of heart notably diminishes, which exacerbates existing abnormalities (Fig. 2) (18).

As TCs are vital to intercellular signalling in human heart, decrease in their number may lead to important disturbances in bioelectrical communication and contribute in arrhythmogenesis (15). Moreover, TCs were found in myocardial sleeves of pulmonary veins, which are considered a source of ectopic beat in atrial fibrillation, so TCs may be related with its pathogenesis (32). Another theory connected with putative origins of atrial fibrillation is isolated atrial amyloidosis existence, but it still has not been established if this condition is a cause or a consequence of atrial fibrillation. Nevertheless, there is a strong association between the presence of amyloidosis in atrial tissue and TCs, as thanks to their long extensions, they surround and gather amyloid fibrils. Based on those findings, it is supposed that TCs are involved in amyloid deposits formation in isolated atrial amyloidosis (32).

There are also some pathological states, which are connected with increased TCs density in heart tissue. The first example is compensated left ventricular hypertrophy, where TCs play a supporting role in maintaining properly working three-dimensional network in myocardium of increased thickness (18). Moreover, there is a positive correlation between the number of TCs in heart tissue and the number of denatured collagens or non-fibrillar collagens, as well as with advancement of acute or chronic myocardial inflammation (6). There exists an assumption that CD34+ fibroblasts, with their morphology congruent to TCs, are important for pathogenesis of myxomatous valve degeneration, as their number is elevated in this condition in comparison to normal, unaffected valves (15).

\section{Current achievements of TCs-related experimental therapies}

As regenerative and anti-remodelling potential of cardiac TCs was described, it became obvious that the next step is making use of this knowledge in clinical practise (18). Zhao et al, as precursors of studies at that issue, transplanted cardiac TCs intramyocardially within 30 minutes after the induced MI (3 injections in a border zone of MI, 2 injections in the centre of an ischaemic area). The results were assessed 14 weeks after MI. Surprisingly, transplantation of TCs into the infarcted myocardium led to the decrease of the affected area, enhanced myocardial function (higher left ventricle ejection fraction), lowered final diastolic diameters and final systolic diameters (compared to control group) and increased density of TCs in infarct zone. Furthermore, angiogenesis was augmented and normal reconstruction of left ventricle, without excessive fibrosis, was stimulated (observed higher thickness of left ventricle in ischaemic zone and in border zone as well as lower collagen area in infarct zone compared to control group) (42). Thus, there exists a potential to reverse, at least partially, MI consequences.
Another experimental therapy aimed at alleviating MI effects is the transplantation of human induced pluripotent stem cell-derived cardiac progenitors (hiPSC-derived CP) or hiPSC-derived mesenchymal stem cells (IMSCs) into MI region, as Ja et al and Miao et al did. Despite the fact that this method seems to be unrelated to TCs, it was observed that 4 weeks after transplantation endogenous and exogenous cardiac TCs appeared and intermingled in the infarction zone. What is the most important is the fact that recovery of their network in ischaemic area coincided with increased angiogenesis and improvement of myocardial strain $(43,44)$.

\section{Vascular telocytes}

\section{Cell morphology and distribution in vascular system}

Generally, vascular TCs seem to have a typical morphology, with some exceptions $(45,46)$. Cell bodies of TCs located in aortic arch were smaller (range from $6.06 \mu \mathrm{m}$ to $13.02 \mu \mathrm{m}$ in length and from $1.05 \mu \mathrm{m}$ to $4.25 \mu \mathrm{m}$ in width), with high nuclear/cytoplasm ratio (47). Moreover, Cantarero et al reported a unique feature of vascular TCs, namely a primary cilium. This immotile cilium contains $9+0$ axoneme, as opposed to $9+2$ axoneme of majority of motile cilia (45).

As for the location, TCs were discovered in almost all layers of blood vessels - except for tunica media (47). In tunica adventitia, cell bodies of TCs are arranged parallelly to the longitudinal axis of the vessel, extending their Tps to form a convoluted network $(45,47)$. In innermost part of intima, TCs exist either in groups or as a single cell, occasionally extending their Tps into the lumen of the vessel (46). Presumably, due to their small tolerance to turbulent blood flow, TCs adherent to endothelial layer were found only in medium-sized arteries (46).

\section{Markers of vascular TCs}

So far, immunohistochemical markers used for identification of vascular TCs include CD34, vimentin and PDGFR- $\beta(17,45)$. Expression of c-kit (CD117), along with Nanog and Sca-1 (which are histochemical markers characteristic for stem cells) was also confirmed in vascular telocytes (17). However, detailed antigen profile of vascular TCs, especially depending on their developmental stage, is yet to be described.

Moreover, expression of angiogenic VEGF and NOS2 was observed in the border zone of MI in TCs located in the close proximity to capillaries (41). Additionally, cytochemical analysis of TCs located in the muscular stem cell niche demonstrated expression of endoglin (CD105), which is a vascular proliferation marker (48).

\section{Intercellular junctions}

TCs form an interstitial network surrounding blood vessels. They are connected with each other through gap junctions or desmosomes $(45,47,49)$. TCs accompanying capillaries frequently establish heterocellular contacts with pericytes and endothelial cells. Those junctions were initially described as planar or point contacts, since no classic electron dense structures were found (24). No evidence has been found of direct connections between TCs and vascular smooth muscle cells (VSMCs) (47). 


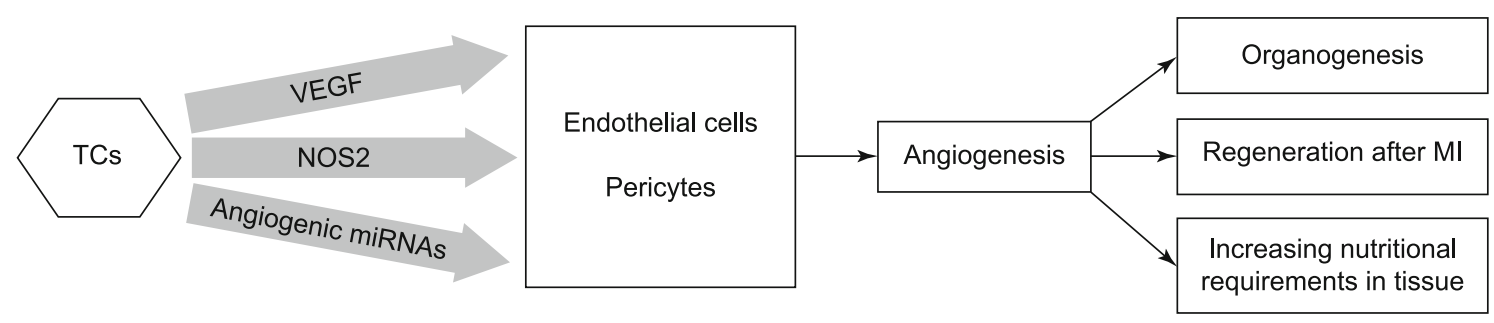

Fig. 3. The role of telocytes (TCs) in angiogenesis, MI - myocardial infarction.

\section{Physiological role of vascular TCs}

Physiological role of vascular TCs is associated with their ability to form convoluted network, similar to the neural one, which is vital for signal integration from numerous systems (5). Occasionally, TCs express miRNAs specific for cells of different type (such as miR-143/145 characteristic for VSMCs), which may indicate their participation in convergence of many complex signalling pathways (41).

TCs concentrated around vasculature may have an ability to control blood flow. Such phenomenon was observed in human term placenta, where TCs form a network around myofibroblasts and VSMCs. This regulatory mechanism may include NO signalisation, since some of the network-forming TCs expressed iNOS (49).

Another distinctive feature of TCs is the ability to promote angiogenesis, thanks to intercellular contacts with endothelial cells and pericytes together with secretion of VEGF, NOS2 and angiogenic miRNAs (e.g. miR-126, miR-130a, let-7 family, miR-10, miR-155, miR-503) (50). It is critical not only for the organogenesis, but also for regeneration after MI $(38,50)$. Furthermore, TCs facilitate formation of abundant vascular network in tissues of high nutritional requirements such as skeletal muscles (Fig. 3) (48).

Additionally, TCs promote and assist vascular regeneration. Incubation of VSMCs in supernatant of vascular TCs resulted in their increased proliferation rate, whereas incubation in supernatant containing TCs pre-treated with antagomir- 24 caused a decline in a proliferation rate. MiR-24, secreted by vascular TCs, was found to be the key regulator of VSMCs proliferation, occurring during vascular injury repair. Another factor influencing vascular regeneration is VEGF, which has a supplementary role in VSMCs proliferation. Different kinds of miRNAs such as miR-221 and miR146a may also affect these processes, however, currently there is no evidence supporting the involvement of TCs in secretion of above mentioned molecules (17). Although, the role of TCs in vascular repair is still not clearly described.

\section{Potential clinical role of TCs in vascular system}

Vascular injury leads to excessive proliferation of TCs, eventually leading to formation of neointima, which may result in re-stenosis of the vessel (17). Owing to that fact, further studies concentrated on functions of TCs in pathophysiological vascular repair may help control various diseases deriving from vascular injury and VSMCs hyperplasia, for example atherosclerosis (17).
Since it has been confirmed that TCs play an important role in supporting angiogenesis in tissue, their properties may be valuable in clinical work. Their physiological ability to facilitate creation of stable vasculature in growing or regenerating tissues should be transferred into synthetically created ones, which can be a milestone in tissue engineering (33).

\section{Glimpse into the future}

TCs present a particular interstitial cell phenotype, which can be more clearly identified and characterized at the ultrastructural level. Despite the wide usage of TEM and CD34 marker in distinguishing TCs from other similar cells, there is still a need for a universal marker. To be reliable (especially for cardiac TCs), it should be indicated throughout the whole cell life - from the embryonic stage until death. Getting further into the subject, there might emerge a seek for subpopulations of TCs in heart and blood vessels, which might be responsible for different diseases.

So far, the studies on TCs have been conducted based on the morphology, whereas it is necessary to prove the direct role of them in the both physiological and pathological conditions. To make it happen, there ought to be more functional studies e.g. transplantation of TCs (gain-of-function assay) or specific cell inhibition (loss-of-function assay) not only in in vitro conditions, but also on animal, in vivo models. It would be vital to establish co-culture of TCs with other cells, for example endogenous CSCs, and observe the impact on the creation of three-dimensional cellular network, as well as a secondary effect of CSCs on TCs. It would help unravel and state the way that TCs support the embryogenesis of heart and the regeneration process of cardiac muscle. Thus, there would be an open door for the enhancement of organ printing technology (51).

A clear direction is presented by comparative anatomy. The new heart is a role model for heart regeneration, since it can functionally regenerate its heart after amputation of the ventricle apex (6). An important aim for regenerative medicine is to find factors to stimulate autologous TCs in situ.

TCs are potentially useful in the future treatment of MI. Not only, as it was mentioned above, would they stimulate CSCs and prevent fibrosis of ischemic area, but also, due to high concentration of SOD2 they would help in reducing the oxidative destruction of a muscle after reperfusion. With their mechanoelectrical potential, TCs might occur as a potentially new player in the 
treatment of heart arrhythmia (following their contribution into the pathomechanism of atrial fibrillation).

Since TCs are proved to regulate the process of inflammation, and furthermore fibrosis, they might be useful in the management of series of disorders connected with heart failure e.g. myocarditis, cardiomyopathy, valve insufficiency itself and related to 33fibrosis.

Angiogenesis provoked by the affection of cardiac TCs might be used in the development of cancer treatment. Some tumours, which represent weak vascularisation are hard to reach by conventional chemotherapeutic drugs. Its enhancement would help in more effective management process. On the other hand, a large network of blood vessels within the tumour promotes metastases. In this case, the effect of TCs on VSMCs' proliferation with a subsequent production of neointima in vasculature and finally vessel stenosis would help in limiting the level of blood flow through the tumour. The balance between proper vascularisation and limitation of blood amount is a key for the success of cancer treatment (52).

Numerous forms of cellular signalling, as well as the multiplicity of excreted molecules and their effect on immune cells can make TCs a future contributor in search for the new pathways of cancer handling and drug delivery (53).

Regenerative function of cardiac TCs in the model of MI is now being widely investigated. They might be useful not only in cases of uncomplicated patients, but also in myocardial or papillary muscle rupture, as an accompanying method to cardiosurgical intervention (through restoration of cellular signalling pathways, as a kind of a tissue glue).

\section{Conclusions}

TCs are unique cell population, which is vital for regeneration, angiogenesis and intercellular signalling in many organs and systems, for instance, in the cardiovascular system. They are presumably connected with pathogenesis of numerous cardiovascular diseases (for instance myocardial infarction) and further experiments involving them may lead to a turning point in treatment of those common conditions.

\section{References}

1. Popescu LM, Faussone-Pellegrini MS. TELOCYTES - a case of serendipity: the winding way from Interstitial Cells of Cajal (ICC), via Interstitial Cajal-Like Cells (ICLC) to TELOCYTES. J Cell Mol Med 2010; 14 (4): 729-740.

2. Faussone Pellegrini MS, Cortesini C, Romagnoli P. Ultrastructure of the tunica muscularis of the cardial portion of the human esophagus and stomach, with special reference to the so-called Cajal's interstitial cells. Arch Ital Anat Embriol 1977; 82 (2): 157-177.

3. Gherghiceanu M, Popescu LM. Interstitial Cajal-like cells (ICLC) in human resting mammary gland stroma. Transmission electron microscope (TEM) identification. J Cell Mol Med 2005; 9 (4): 893-910.

4. Pieri L, Vannucchi MG, Faussone-Pellegrini MS. Histochemical and ultrastructural characteristics of an interstitial cell type different from ICC and resident in the muscle coat of human gut. J Cell Mol Med 2008; 12 (5B): 1944-1955.
5. Mirancea N. Telocyte - a particular cell phenotype. Infrastructure, relationships and putative functions. Rom J Morphol Embryol 2016; 57 (1): 7-21.

6. Kostin S. Myocardial telocytes: a specific new cellular entity. J Cell Mol Med 2010; 14 (7): 1917-1921.

7. Li YY, Zhang S, Li YG, Wang Y. Isolation, culture, purification and ultrastructural investigation of cardiac telocytes. Mol Med Rep 2016; 14 (2): 1194-1200

8. Cretoiu SM, Popescu LM. Telocytes revisited. Biomol Concepts 2014; 5 (5): 353-369.

9. Rusu MC, Loreto C, Mănoiu VS. Network of telocytes in the temporomandibular joint disc of rats. Acta Histochem 2014; 116 (4): 663-668.

10. Popescu LM, Manole CG, Gherghiceanu M et al. Telocytes in human epicardium. J Cell Mol Med 2010; 14 (8): 2085-2093.

11. Bei Y, Zhou Q, Sun Q, Xiao J. Telocytes in cardiac regeneration and repair. Semin Cell Dev Biol 2016; 55: 14-21.

12. Díaz-Flores L, Gutiérrez R, Goméz MG, Sáez FJ, Madrid JF. Behaviour of telocytes during physiopathological activation. Semin Cell Dev Biol 2016; 55: 50-61.

13. Chang Y, Li C, Lu Z, Li H, Guo Z. Multiple immunophenotypes of cardiac telocytes. Exp Cell Res 2015; 338 (2): 239-244.

14. Faussone-Pellegrini MS, Gherghiceanu M. Telocyte's contacts. Semin Cell Dev Biol 2016; 55: 3-8.

15. Ibba-Manneschi L, Rosa I, Manetti M. Telocyte implications in human pathology: An overview. Semin Cell Dev Biol 2016; 55: 62-69.

16. Faussone-Pellegrini MS, Bani D. Relationships between telocytes and cardiomyocytes during pre- and post-natal life. J Cell Mol Med 2010; 14 (5): 1061-1063.

17. Li Y, Zhang X, Gao J, Xiao H, Xu M. Increased telocytes involved in the proliferation of vascular smooth muscle cells in rat carotid artery balloon injury. Sci China Life Sci 2016; 59 (7): 678-685.

18. Kostin S. Cardiac telocytes in normal and diseased hearts. Semin Cell Dev Biol 2016; 55: 22-30.

19. Bei Y, Zhou Q, Fu S et al. Cardiac telocytes and fibroblasts in primary culture: different morphologies and immunophenotypes. PLoS One 2015; 10 (2): e0115991.

20. Gerlach JC, Over P, Turner ME et al. Perivascular mesenchymal progenitors in human fetal and adult liver. Stem Cells Dev 2012; 21 (18): 3258-3269.

21. Miettinen M, Lasota J. KIT (CD117): a review on expression in normal and neoplastic tissues, and mutations and their clinicopathologic correlation. Appl Immunohistochem Mol Morphol 2005; 13 (3): 205-220.

22. Cismasiu VB, Radu E, Popescu LM. miR-193 expression differentiates telocytes from other stromal cells. J Cell Mol Med 2011; 15 (5): 1071-1074.

23. Cretoiu SM. Telocytes in focus. Semin Cell Dev Biol 2016; 55: 1-2.

24. Gherghiceanu M, Popescu LM. Cardiac telocytes - their junctions and functional implications. Cell Tissue Res 2012; 348 (2): 265-279.

25. Edelstein L, Fuxe K, Levin M, Popescu BO, Smythies J. Telocytes in their context with other intercellular communication agents. Semin Cell Dev Biol 2016; 55: 9-13. 
26. Popescu LM, Fertig ET, Gherghiceanu M. Reaching out: junctions between cardiac telocytes and cardiac stem cells in culture. J Cell Mol Med 2016; 20 (2): 370-380.

27. Fertig ET, Gherghiceanu M, Popescu LM. Extracellular vesicles release by cardiac telocytes: electron microscopy and electron tomography. J Cell Mol Med 2014; 18 (10): 1938-1943.

28. Edelstein L, Smythies J. The role of telocytes in morphogenetic bioelectrical signaling: once more unto the breach. Front Mol Neurosci 2014; 7: 41 .

29. Popescu LM, Curici A, Wang E, Zhang H, Hu S, Gherghiceanu M. Telocytes and putative stem cells in ageing human heart. J Cell Mol Med 2015; 19 (1): 31-45.

30. Richter M, Kostin S. The failing human heart is characterized by decreased numbers of telocytes as result of apoptosis and altered extracellular matrix composition. J Cell Mol Med 2015; 19 (11): 2597-2606.

31. Gherghiceanu M, Manole CG, Popescu LM. Telocytes in endocardium: electron microscope evidence. J Cell Mol Med 2010; 14 (9): 2330-2334.

32. Gherghiceanu M, Hinescu ME, Andrei F et al. Interstitial Cajal-like cells (ICLC) in myocardial sleeves of human pulmonary veins. J Cell Mol Med 2008; 12 (5A): 1777-1781.

33. Boos AM, Weigand A, Brodbeck R, Beier JP, Arkudas A, Horch RE. The potential role of telocytes in Tissue Engineering and Regenerative Medicine. Semin Cell Dev Biol 2016; 55: 70-78.

34. Gherghiceanu M, Popescu LM. Cardiomyocyte precursors and telocytes in epicardial stem cell niche: electron microscope images. J Cell Mol Med 2010; 14 (4): 871-877.

35. Yang Y, Sun W, Wu SM, Xiao J, Kong X. Telocytes in human heart valves. J Cell Mol Med 2014; 18 (5): 759-765.

36. Cismasiu VB, Popescu LM. Telocytes transfer extracellular vesicles loaded with microRNAs to stem cells. J Cell Mol Med 2015; 19 (2): 351-358.

37. Albulescu R, Tanase C, Codrici E, Popescu DI, Cretoiu SM, Popescu LM. The secretome of myocardial telocytes modulates the activity of cardiac stem cells. J Cell Mol Med 2015; 19 (8): 1783-1794.

38. Xiao J, Chen P, Qu Y et al. Telocytes in exercise-induced cardiac growth. J Cell Mol Med 2016; 20 (5): 973-979.

39. Enciu AM, Popescu LM. Telopodes of telocytes are influenced in vitro by redox conditions and ageing. Mol Cell Biochem 2015; 410 (1-2): $165-174$.
40. Sheng J, Shim W, Lu J et al. Electrophysiology of human cardiac atrial and ventricular telocytes. J Cell Mol Med 2014; 18 (2): 355-362.

41. Zhao B, Chen S, Liu J et al. Cardiac telocytes were decreased during myocardial infarction and their therapeutic effects for ischaemic heart in rat. J Cell Mol Med 2013; 17 (1): 123-133.

42. Zhao B, Liao Z, Chen S et al. Intramyocardial transplantation of cardiac telocytes decreases myocardial infarction and improves post-infarcted cardiac function in rats. J Cell Mol Med 2014; 18 (5): 780-789.

43. Ja KP, Miao Q, Zhen Tee NG et al. iPSC-derived human cardiac progenitor cells improve ventricular remodelling via angiogenesis and interstitial networking of infarcted myocardium. J Cell Mol Med 2016; 20 (2): 323-332.

44. Miao Q, Shim W, Tee $\mathbf{N}$ et al. iPSC-derived human mesenchymal stem cells improve myocardial strain of infarcted myocardium. J Cell Mol Med 2014; 18 (8): 1644-1654.

45. Cantarero I, Luesma MJ, Junquera C. The primary cilium of telocytes in the vasculature: electron microscope imaging. J Cell Mol Med 2011; 15 (12): 2594-2600.

46. Li H, Lu S, Liu H, Ge J, Zhang H. Scanning electron microscope evidence of telocytes in vasculature. J Cell Mol Med 2014; 18 (7): 1486-1489.

47. Zhang HQ, Lu SS, Xu T, Feng YL, Li H, Ge JB. Morphological evidence of telocytes in mice aorta. Chin Med J (Engl) 2015; 128 (3): 348-352.

48. Bojin FM, Gavriliuc OI, Cristea MI et al. Telocytes within human skeletal muscle stem cell niche. J Cell Mol Med 2011; 15 (10): 2269-2272.

49. Suciu L, Popescu LM, Gherghiceanu M et al. Telocytes in human term placenta: morphology and phenotype. Cells Tissues Organs 2010; 192 (5): 325-339.

50. Manole CG, Cismasiu V, Gherghiceanu M, Popescu LM. Experimental acute myocardial infarction: telocytes involvement in neo-angiogenesis. J Cell Mol Med 2011; 15 (11): 2284-2296.

51. Murphy SV, Atala A. 3D bioprinting of tissues and organs. Nat Biotechnol 2014; 32 (8): 773-785.

52. Nishida N, Yano H, Nishida T, Kamura T, Kojiro M. Angiogenesis in cancer. Vasc Health Risk Manag 2006; 2 (3): 213-219.

53. Eyileten C, Majchrzak K, Pilch $\mathbf{Z}$ et al. Immune Cells in Cancer Therapy and Drug Delivery. Mediators Inflamm 2016; 2016:5230219.

Received January 12, 2017. Accepted January 23, 2017. 